\title{
Redistribution métabolique chez une souche de Lactobacillus plantarum déficiente en lactate déshydrogénase
}

\author{
Thierry Ferain ${ }^{a}$, André N. Schanck ${ }^{b}$, Jeroen Hugenholtz ${ }^{c}$, \\ Pascal Hols ${ }^{\text {a }}$, Willem M. de Vos ${ }^{d}$, Jean Delcour ${ }^{a}$
}

${ }^{a}$ Laboratoire de génétique moléculaire, unité de génétique, université catholique de Louvain, 1348 Louvain-la-Neuve, Belgique

${ }^{b}$ Laboratoire de chimie physique et de cristallographie, université catholique de Louvain, 1348 Louvain-la-Neuve, Belgique

' Departments of microbiology, NIZO, Ede, Pays-Bas

${ }^{d}$ Departments of biophysical chemistry, NIZO, Ede, Pays-Bas

\begin{abstract}
Metabolic rerouting in a Lactobacillus plantarum strain deficient for lactate dehydrogenase. We constructed a strain of Lactobacillus plantarum deficient for both D- and L-lactate dehydrogenase activities by gene inactivation. The metabolism of the resulting strain, which lacks its main fermentation pathway, was analysed by nuclear magnetic resonance. This study showed a major rerouting of both glucose and glucose/citrate metabolism which led to the production of end-products such as acetoin, ethanol, acetate, mannitol, succinate, etc. Following the heterologous expression of $a d h$ and $p d c$ genes from Zymomonas mobilis in this strain, a part of the pyruvate flux was channeled to the production of ethanol. (c) Inra/Elsevier, Paris.
\end{abstract}

Lactobacillus plantarum / lactate dehydrogenase / metabolism / Zymomonas mobilis / ethanol

Résumé - Nous avons construit une souche de Lactobacillus plantarum dont les gènes codant pour les deux activités lactate déshydrogénase ont été inactivés, privant de cette manière la bactérie de sa principale voie de fermentation. L'analyse de cette souche mutante par résonance magnétique nucléaire a permis de mettre en évidence la réorientation complète du métabolisme du glucose, ainsi que du cométabolisme glucose/citrate, vers la production de composés tels que l'acétoïne, l'éthanol, l'acétate, le mannitol, le succinate... L'expression hétérologue des gènes $p d c$ et $a d h$ de Zymomonas mobilis a provoqué chez cette souche une réorientation partielle du flux de pyruvate vers la production d'éthanol. (C) Inra/Elsevier, Paris.

Lactobacillus plantarum / lactate déshydrogénase / métabolisme / Zymomonas mobilis / éthanol 


\section{INTRODUCTION}

Les bactéries lactiques doivent leur nom à cette particularité métabolique de fermenter les sucres principalement en acide lactique. Cette dénomination peut donner l'impression d'un métabolisme rigide, peu flexible, laissant percevoir les bactéries lactiques comme limitées à cette conversion des sucres en lactate. On sait pourtant que ce n'est pas le cas. Même si le lactate est souvent le produit majeur de fermentation, d'autres métabolites peuvent également être synthétisés lors du métabolisme des sucres. En réalité, l'apparition du lactate comme composé majeur correspond souvent à une croissance sur un excès de glucose, l'accumulation massive de lactate étant principalement le reflet de la phase exponentielle de croissance. Lorsque l'on fait varier certains paramètres de culture tels la disponibilité en oxygène, la nature et la quantité de la source de carbone ou encore le $\mathrm{pH}$, on observe fréquemment différentes réorientations métaboliques.

Chez Lactobacillus plantarum, bactérie lactique à laquelle est consacrée cette étude, la fermentation lactique emprunte dans un premier temps la glycolyse. Le pyruvate qui en résulte est ensuite réduit en lactate de forme $\mathrm{D}$ et $\mathrm{L}$ grâce à l'activité de deux lactate déshydrogénases (LDH) distinctes et stéréospécifiques, avec réoxydation simultanée du cofacteur NADH en $\mathrm{NAD}^{+}$. Il existe néanmoins d'autres voies de dissipation du pyruvate (figure 1) qui donnent lieu à la formation de composés tels l'acétate, l'éthanol, l'acétoïne, le 2,3-butanediol, le formate... La participation de ces différentes voies dans le flux fermentaire sera fonction des différents paramètres physicochimiques caractérisant l'environnement bactérien.

L'objectif de notre travail a été dans un premier temps de construire une souche de L. plantarum déficiente en activité lactate déshydrogénase afin d'étudier la réor- ganisation du métabolisme responsable de la dissipation du pyruvate.

Dans un second temps, nous avons évalué la possibilité de réorienter le métabolisme fermentaire de cette souche vers la production d'un composé choisi grâce à l'expression hétérologue de gènes métaboliques originaires de Zymomonas mobilis, bactérie à Gram négatif dont le métabolisme est spécialisé dans la production d'éthanol. L'idée d'une conversion de sucres en éthanol par voie de fermentation n'est pas un nouveau concept en milieu industriel, et plusieurs espèces et souches de levures sont déjà largement exploitées dans ce sens. Z. mobilis ellemême est un candidat intéressant, ayant un métabolisme naturellement focalisé vers la production d'éthanol. L'intérêt porté aux bactéries lactiques dans ce domaine, et dans notre cas plus spécifiquement à L. plantarum, trouve son origine dans plusieurs avantages que pourraient présenter certaines espèces appartenant à ce groupe.

D'abord, la gamme de substrats métabolisables par bon nombre de bactéries lactiques est particulièrement large en comparaison à celle accessible aux levures et à Z. mobilis [21]. En particulier, certains sous-produits des industries laitière et agricole de coût peu élevé (lactose du lactosérum, amidon non liquéfié) pourraient avantageusement être convertis en éthanol par des espèces telles que Lactococcus lactis, Lactobacillus helveticus, Lactobacillus plantarum.

Une seconde caractéristique qui pourrait être avantageusement exploitée consiste en un bon niveau de résistance à l'éthanol chez certaines bactéries lactiques [7]. En effet, l'activité fermentaire des microorganismes en cours de fermentation alcoolique diminue à mesure que la concentration en éthanol augmente dans le milieu extracellulaire. Ce stress à l'éthanol est le résultat de plusieurs facteurs liés 


\section{$1 / 2$ GLUCOSE}

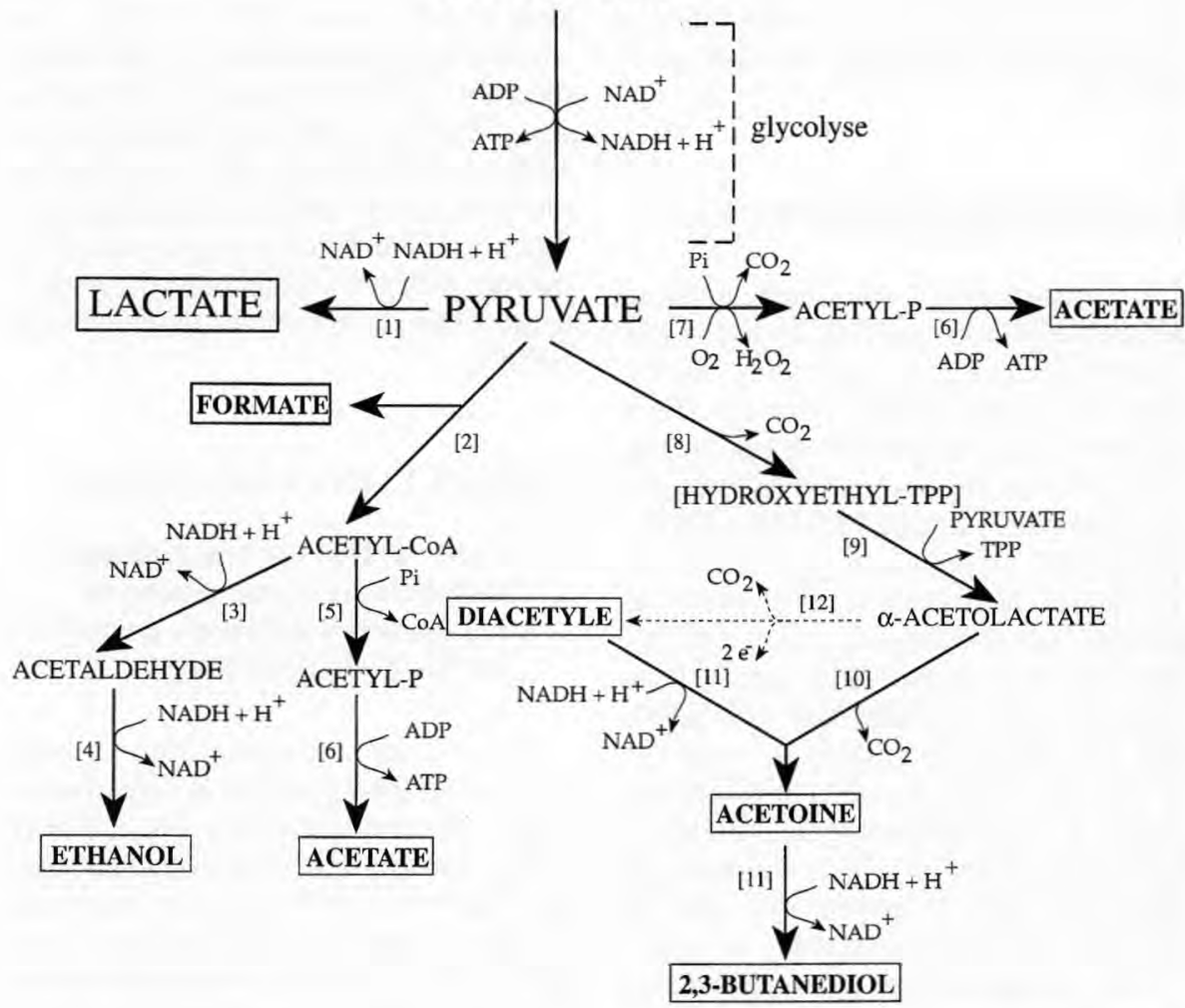

Figure 1. Voies de fermentation du glucose et de dissipation du pyruvate proposées pour la souche NCIMB8826 de Lactobacillus plantarum ; 1 lactate déshydrogénase ; 2 pyruvate formate lyase ; 3 acétaldéhyde déshydrogénase ; 4 alcool déshydrogénase ; 5 phosphotransacétylase ; 6 acétate kinase ; 7 pyruvate oxidase ; 8 pyruvate décarboxylase $; 9 \alpha$-acétolactate synthase ; $10 \alpha$-acétolactate décarboxylase ; 11 2,3-butanediol déshydrogénase ; 12 décarboxylation non enzymatique.

Figure 1. Pathways involved in glucose fermentation and pyruvate dissipation in Lactobacillus plantarum NCIMB8826. 1, lactate dehydrogenase; 2 , pyruvate formate lyase; 3 , acetaldehyde dehydrogenase; 4 , alcohol dehydrogenase; 5 , phosphotransacetylase; 6 , acetate kinase; 7 , pyruvate oxidase; 8 , pyruvate decarboxylase; $9, \alpha$-acetolactate synthase; $10, \alpha$-acetolactate decarboxylase; 11, 2,3-butanediol dehydrogenase; 12, non-enzymatic decarboxylation.

non seulement à un effet néfaste sur les activités enzymatiques (effet dénaturant), mais également une altération majeure au niveau de la membrane cellulaire [12]. Il en résulte un rendement tout à fait acceptable à de faibles concentrations en sucres, mais avec une productivité qui chute considérablement à des concentrations d'éthanol comprises entre 6 et $8 \%$. Bien que n'étant pas toutes tolérantes à de fortes concentrations en éthanol, une étude systématique de quelques espèces de bactéries lactiques à montré que plusieurs d'entre elles, dont certaines souches de L. plan- 
tarum sont caractérisées par une croissance relativement peu affectée par des concentrations en éthanol allant jusqu'à $16 \%$ [7].

\section{CONTEXTE EXPÉRIMENTAL}

La construction d'une souche mutante déficiente pour les activités D- et L-LDH a nécessité dans un premier temps le clonage des gènes codant pour ces deux enzymes. Les stratégies développées dans notre cas ont été basées sur la complémentation de la souche FMJ144 d'Escherichia coli [19] déficiente pour les activités lactate déshydrogénase et pyruvate formate lyse (clonage du gène ldhL [3]), ainsi que sur l'amplification par PCR au départ d'oligonucléotides dégénérés ( $l d h D$, [4]). Les gènes ont ensuite été inactivés in vitro par délétion (ldhL) et disruption $(l d h D)$. Ces altérations ont été individuellement transférées au sein du chromosome bactérien afin d'obtenir une souche mutante entièrement stable, désignée TF103, et caractérisée par sa déficience en activité $\mathrm{LDH}$.

Une étude métabolique de cette souche a été réalisée par résonance magnétique nucléaire basée sur l'abondance naturelle de l'isotope 13 du carbone [5]. Cette étude a été effectuée sur des suspensions cellulaires additionées de glucose $50 \mathrm{mmol} / \mathrm{L}$ ainsi que de citrate $10 \mathrm{mmol} / \mathrm{L}$ dans le cas des études de cométabolisme. Cela nous a permis d'indentifier et de quantifier une variété de composés issus du métabolisme du glucose et du citrate.

Le travail destiné à augmenter la production d'éthanol chez cette souche a mis en oeuvre un opéron créé de manière artificielle dans lequel les gènes codant pour la pyruvate décarboxylase ( $p d c$ ) et l'alcool déshydrogénase $(a d h)$ de Zymomonas mobilis sont sous le contrôle du couple de promoteurs prtM/prtP de Lactococcus lactis [18]. Cette unité de transcription a été introduite sur un vecteur autoréplicatif chez L. plantarum (pGK13, [15]). Une caractérisation préliminaire de cette souche recombinante a été réalisée en fermentation « batch » à $\mathrm{pH}$ fixe (régulation par addition de $\mathrm{NaOH}$ ou $\mathrm{HCl} 2 \mathrm{~N}$ ) dans un milieu de culture MRS (de Man, Rogosa et Sharpe). L'identification et la quantification des différents métabolites issus de la dégradation du glucose a été réalisée par HPLC.

\section{RÉSULTATS ET DISCUSSION}

\subsection{Construction et caractérisation métabolique d'une souche de L. plantarum déficiente en activité lactate déshydrogénase}

Le clonage des gènes $l d h L$ et $l d h D$ de $L$. plantarum a permis la construction d'une souche inactivée pour ces deux gènes. La stratégie d'inactivation, bien que légèrement différente pour chacun de ces gènes, peut être schématisée selon la figure 2. Il s'agit d'un processus de recombinaison homologue en deux étapes (intégration/excision) dont l'aboutissement résulte en une délétion interne pour le gène ldhL [3], et en une disruption par un marqueur de résistance au chloramphénicol (gène cat) pour le gène $l d h D$ [4]. Ce type de stratégie garantit une entière stabilité des altérations chez cette souche, désignée TF103, qui par ailleurs ne présente plus d'activité LDH,

La caractérisation métabolique de cette souche par résonance magnétique nucléaire a été réalisée en comparaison avec la souche parentale NCIMB8826 [5]. Lorsque les supsensions cellulaires sont incubées en présence d'oxygène (figure $3 A$ ), le métabolisme du glucose se caractérise par une distribution simple chez la souche sauvage: une production majoritaire de lactate, accompagnée d'un faible pourcentage d'acétate. Par contre, la souche TF103 présente un profil métabolique nettement 


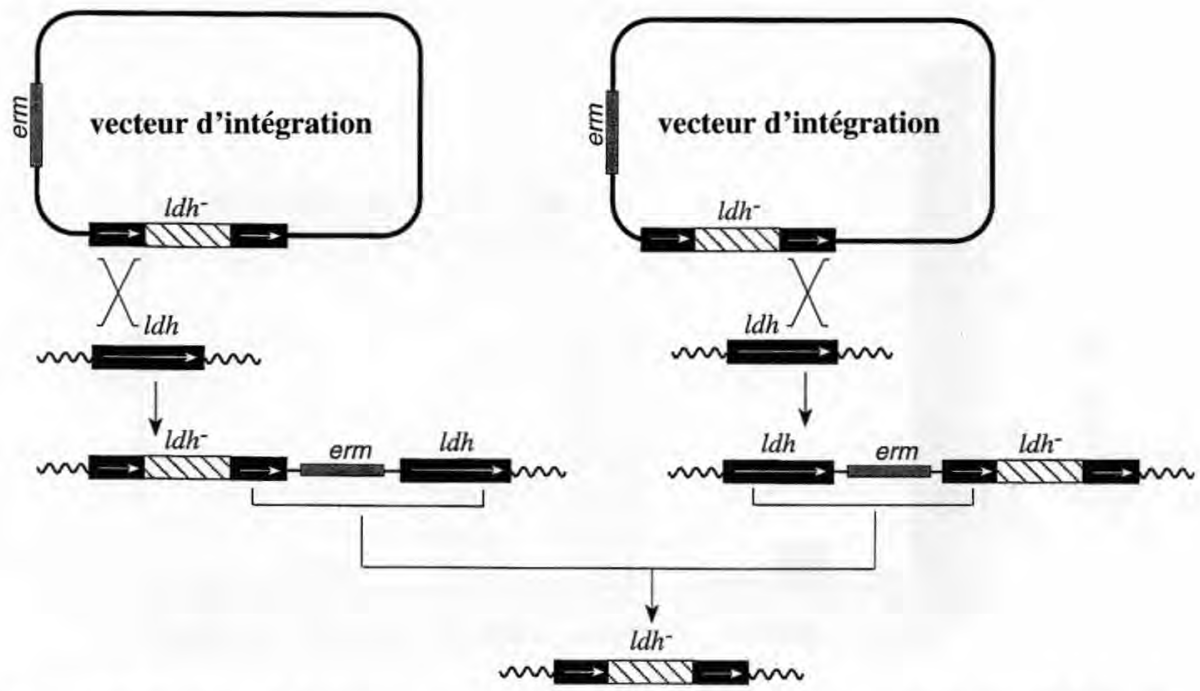

Figure 2. Stratégie générale mise en œuvre pour l'inactivation des gènes $l d h L$ et $l d h D$ de $L$. plantarum. Une copie plasmidique du gène cible est inactivée in vitro par délétion (gène $l d h L$ ) ou par disruption (gène $l d h D$ ), donnant lieu à un gène non fonctionnel désigné $l d h$ sur la figure. Un processus de recombinaison homologue en deux étapes (intégration/excision) permet le transplacement stable de cette altération au sein du chromosome bactérien.

Figure 2. Strategy developed for $l d h$ genes inactivation: a plasmid-borne copy of the gene is inactivated in vitro by deletion (ldhL gene) or disruption (ldhD gene). A two-step homologous recombination process (integration/excision) leads to a stable inactivation of the chromosomal copy of the gene.

plus diversifié. Le composé majeur est cette fois l'acétoïne, accompagné également d'éthanol, d'acétate, de succinate, de mannitol, ainsi que de faibles quantités de lactate. L'apparition de ce dernier métabolite chez la souche TF103 peut paraître surprenante, compte tenu de l'absence d'activité LDH. Il est néanmoins possible que des activités enzymatiques apparentées, connues sont le nom d'hydroxyisocaproate déshydrogénase, soient à l'origine d'une faible conversion du pyruvate en lactate $[2,16,17]$. Bien que ce type d'activité ait été mis en évidence chez certaines espèces de bactéries lactiques, son existence chez notre souche de $L$. plantarum reste purement spéculative.

Une caractérisation métabolique similaire a également été réalisée en conditions anaérobies en incubant les sus- pensions cellulaires sous flux d'azote (figure 3B). Dans ce cas, la souche mutante TF103 est incapable de métaboliser le glucose seul. Cette défícience est vraisemblablement liée à une incapacité de réoxyder le cofacteur NADH formé au cours de la glycolyse. En aérobiose, ce recyclage indispensable serait assuré par le système NADH oxidase/peroxidase [9]) dont le fonctionnement exige la présence d'oxygène moléculaire. Par contre, en anaérobiose, il semblerait que l'équilibre rédox intracellulaire ne puisse pas être maintenu, probablement suite à une régénération partielle du $\mathrm{NAD}^{+}$par les voies schématisées à la figure 1. Cependant, l'addition d'un accepteur d'électrons externé tel que le citrate en plus du glucose donne lieu au cométabolisme de ces deux substrats. En effet, le citrate permet un apport supplé- 

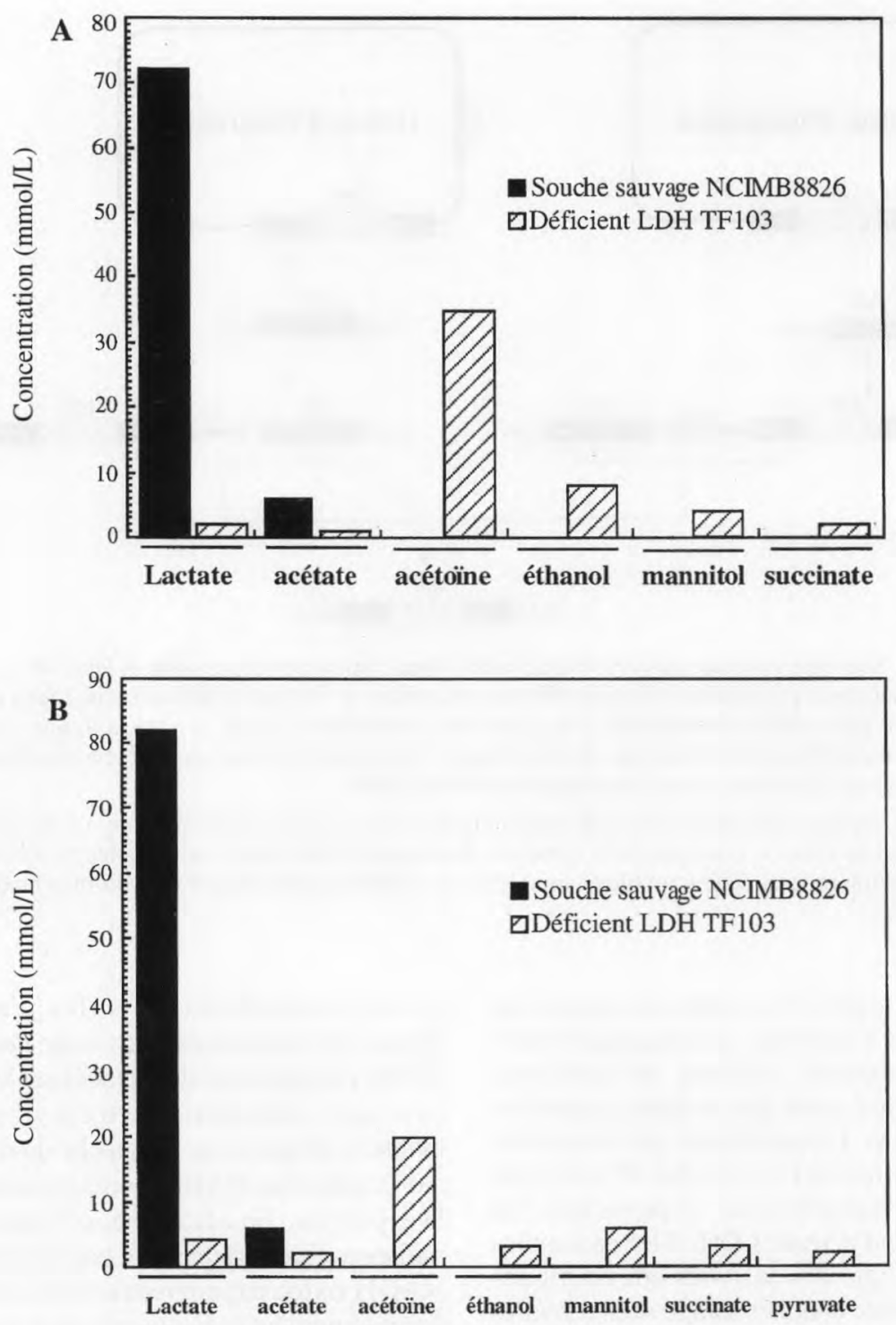

Figure 3. Produits du métabolisme du glucose et du cométabolisme glucose/citrate par des suspensions cellulaires des souches sauvages NCIMB8826 et mutante TF103 de L. plantarum. Les supsensions cellulaires ont été incubées dans les conditions suivantes. A. Glucose $50 \mathrm{mmol} / \mathrm{L}$ sous flux d'air (conditions aérobies). B. Glucose $50 \mathrm{mmol} / \mathrm{L}^{+}$citrate $10 \mathrm{mmol} / \mathrm{L}$ sous flux d'azote (conditions anaérobies). Les produits de fermentation ont été identifiés par résonance magnétique nucléaire.

Figure 3. End-products from glucose metabolism and glucose/citrate co-metabolism in the wildtype strain NCIMB8826 and the mutant strain TF103. Studies have been performed using cell suspensions incubated in the following conditions. A. Glucose $50 \mathrm{mmol} / \mathrm{L}$, aerobiosis. B. Glucose $50 \mathrm{mmol} / \mathrm{L}^{+}$citrate $10 \mathrm{mmol} / \mathrm{L}$, anaerobiosis. End-products have been identified by ${ }^{13} \mathrm{C}$ nuclear magnetic resonance. 
mentaire de pyruvate sans passer par la voie de la glycolyse génératrice de NADH [11]. Ce pyruvate, dirigé dans une certaine mesure vers des voies productrices de $\mathrm{NAD}^{+}$, rétablirait l'équilibre redox. L'analyse de ce cométabolisme glucose/citrate entraîne la production de lactate et d'acétate chez la souche sauvage. Chez la souche mutante, l'acétoïne reste le catabolite majeur, accompagné des composés déjà observés lors de l'incubation aérobie ainsi que d'une faible concentration en pyruvate.

\subsection{Expression hétérologue des gènes pdc et adh de Zymomonas mobilis chez L. plantarum TF103}

La caractérisation métabolique de la souche TF103 nous a amené à penser que le niveau de pyruvate intracellulaire était élevé chez ce mutant. En effet, l'activation de la voie conduisant à l'acétoïne est caractéristique d'une haute concentration intracellulaire en pyruvate [22]. De plus, l'apparition de pyruvate dans le milieu extracellulaire est également en faveur d'un engorgement de ce métabolite [10,23]. Il semblait dès lors possible de rediriger cet excès de pyruvate vers la production massive d'un composé choisi, que ce soit dans une optique industrielle, ou simplement pour démontrer la faisabilité d'une telle stratégie d'ingénierie métabolique chez cette bactérie lactique. Parmi différentes possibilités, la production d'éthanol au départ du pyruvate a été envisagée grâce à l'intervention de deux enzymes : la pyruvate décarboxylase (PDC) et l'alcool déshydrogénase (ADH) originaires de Zymomonas mobilis. Ces activités sont responsables de la conversion du pyruvate en éthanol selon une séquence en deux étapes dont la seconde fait intervenir la réoxidation d'un équivalant NADH.

L'expression hétérologue des gènes $p d c$ et adh de Z. mobilis chez la souche LDH négative de L. plantarum est donc apparue comme intéressante. Ce type d'étude avait déjà été réalisé chez $E$. coli $[1,13,14$, 20]. Malgré un très bon rendement, cet organisme présente une limitation évidente dans la tolérance à l'éthanol. D'autre part, Lactobacillus casei a également été testé dans une stratégie expérimentale similaire [8]. Dans ce cas, la souche hôte pour l'expression hétérologue était une souche sauvage possédant une activité élevée en $\mathrm{LDH}$. Malgré un certain succès, la compétition entre les deux systèmes de dissipation du pyruvate a vraisemblablement amoindri la production d'éthanol en faveur du lactate, et les auteurs suggèrent d'améliorer la stratégie en reproduisant l'expérience avec une souche de $L$. casei déficiente en $\mathrm{LDH}$.

Notre souche TF103 présentait d'emblée cette caractéristique métabolique avantageuse et a donc été utilisée comme hôte pour l'expression des gènes $p d c$ et $a d h$ de Z. mobilis. L'opéron pet ( production of ethanol »), comporte les phases ouvertes de lecture des deux gènes couplées artificiellement en une seule unité de transcription contrôlée par le couple de promoteurs prtM/prtP de Lactococcus lactis [18]. Cet opéron pet a été inséré dans le plasmide pGK13 [15], réplicon fonctionnel chez $L$. plantarum. L'analyse métabolique a ensuite été réalisée sur la souche TF103 possédant cette construction, ainsi que la même souche pourvue du vecteur pGK13 en guise de contrôle. Les fermentations ont été conduites en milieu MRS contenant $2 \%$ de glucose ainsi que du citrate à une concentration de $8 \mathrm{mmol} / \mathrm{L}$. Plusieurs expériences de fermentations «batch » ont été réalisées en faisant varier 2 paramètres : d'une part, le $\mathrm{pH}$ a été successivement fixé à des valeurs de 5, 6 et 7 ; d'autre part, les fermentations ont été réalisées soit sous atmosphère (aérobiose) ou sous flux d'azote (anaérobiose). Les résultats les plus intéressants ont été obtenus lors de fermentations à $\mathrm{pH} 6$ en condi- 


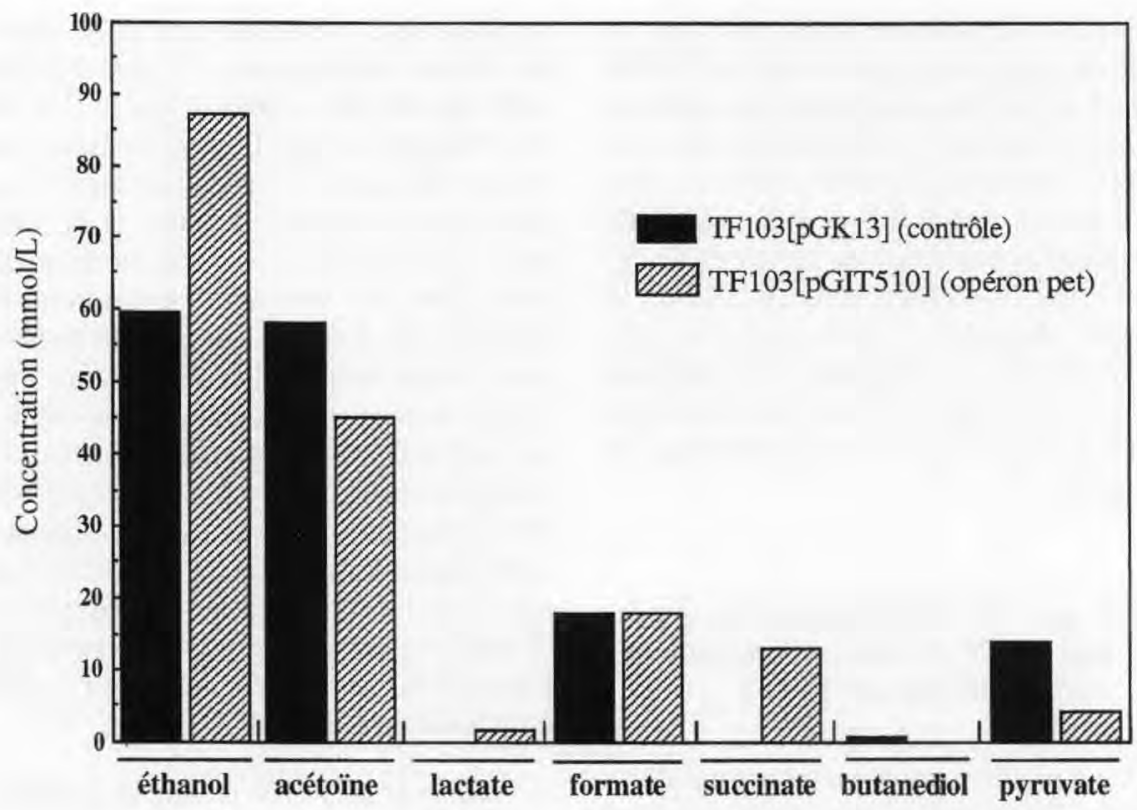

Figure 4. Produits issus de la fermentation en milieu MRS (base glucose $2 \%$ ) de la souche TF103 de $L$. plantarum. Cet histogramme compare un contrôle, qui possède uniquement le vecteur pGK13, avec la même souche dotée du plasmide recombinant pGIT 510 (gènes $p d c$ et $a d h$ ). La fermentation a été conduite sous flux d'azote à pH 6.

Figure 4. Fermentation products from TF103 strain of $L$. plantarum grown in MRS medium (glucose 2\%). This scheme compares TF103 strain bearing a control plasmid (pGK13) and the recombinant ethanol plasmid (pGIT510, $p d c$ and $a d h$ genes). Fermentations were performed at pH 6 under nitrogen stream.

tions anaérobies (figure 4). Dans ce cas, on observe que la présence du plamside recombinant est corrélée à une augmentation de la production d'éthanol chez la souche TF103 avec réduction simultanée du principal métabolite identifié chez cette souche : l'acétoïne. Cependant, la souche contrôle produit également une quantité appréciable d'éthanol, et la réorientation du flux de pyruvate chez la souche recombinante reste encore très partielle. En effet, en ne considérant comme source potentielle d'éthanol que le glucose, on atteint un rendement de $49 \%$ du maximum théorique pour la souche recombinante contre $37 \%$ pour la souche contrôle. Bien que cela n'ait pas été explicité ici, il semble que la présence ou l'absence d'oxygène n'influence pas significativement le rendement de conversion du glucose en éthanol, mais que la cinétique de consommation du glucose soit plus favorable en anaérobiose. Par ailleurs, il apparaît également qu'une valeur basse de $\mathrm{pH}$ (5) favoriseraient la formation d'acétoïne alors qu'une valeur plus élevée (7) ralentirait la formation de biomasse. Un pH de 6 a été présenté ci-dessus comme étant le meilleur compromis actuellement testé.

Il est évident que l'état actuel de ces études constituera une base pour l'amélioration du processus de fermentation alcoolique chez cette souche. Sur le plan 
génétique, l'utilisation du vecteur d'expression dans sa conception actuelle est de toute évidence à l'origine d'un accroissement de la production d'éthanol. Rien n'indique cependant que l'on se situe dans des conditions optimales d'expression des gènes $p d c$ et $a d h$. L'effet limité que nous avons observé pourrait donc simplement refléter une limitation dans les quantités d'enzymes disponibles. Il sera donc nécessaire de tester le niveau d'expression des gènes, et au besoin de l'améliorer en modifiant le système d'expression.

La présence d'autres métabolites du glucose, dont principalement l'acétoïne, pourrait en outre être réduite en faveur de la production d'éthanol. Cela pourrait se faire d'une part en tentant d'optimiser les conditions de fermentations, mais également en envisageant d'autres modifications génétiques de la souche qui, par exemple, inactiveraient la voie de synthèse de l'acétoïne.

Par ailleurs, les quantités de glucose engagées sont encore loin des concentrations réellement mises en cuvre lors de fermentations industrielles. Il faudrait donc voir comment, en présence de quantités plus élevées de glucose, la souche se comporterait en terme de cinétique de consommation, de tolérance réelle à des concentrations élevées en éthanol et en termes de bilan métabolique global. Enfin, un des avantages proposés pour l'utilisation de lactobacilles en production d'éthanol est la large gamme de substrats métabolisable par ce groupe de bactérie. Il sera donc indispensable de tester différents sucres, dont ceux qui présentent un intérêt potentiel au niveau de l'industrie. Nous pensons en particulier profiter des caractéristiques que présente une souche amylolytique de $L$. plantarum capable d'hydrolyser et de fermenter l'amidon brut [6].

\section{RÉFÉRENCES}

[1] Arfman N., Worrell V., Ingram L.O., Use of the $t a c$ promoter and $\operatorname{lacl}^{q}$ for the controlled expression of Zymomonas mobilis fermentative genes in Escherichia coli and Zymomonas mobilis, J. Bacteriol. 174 (1992) 7370-7378.

[2] Bernard N., Johnsen K., Ferain T., Garmyn D., Hols P., Holbrook J.J., NAD+-dependent D-2hydroxyisocaproate dehydrogenase of Lactobacillus delbrueckii subsp bulgaricus. Gene cloning and enzyme characterization, Eur. J. Biochem. 224 (1994) $439-446$.

[3] Ferain T., Garmyn D., Bernard N., Hols P., Delcour J., Lactobacillus plantarum IdhL gene: overexpression and deletion, J. Bacteriol. 176 (1994) 596-601.

[4] Ferain T., Hobbs J.N., Richardson J., Bernard N., Garmyn D., Hols P., Allen N.E., Delcour J., Knockout of the two Idh genes has a major impact on peptidoglycan precursors synthesis in Lactobacillus plantarum. J. Bacteriol. 178 (1996a) 5431-5437.

[5] Ferain T,, Schanck A,N,, Delcour J,, ${ }^{13} \mathrm{C}$ nuclear magnetic resonance analysis of glucose and citrate end products in an ldhL-ldhD double-knockout strain of Lactobacillus plantarum, J. Bacteriol. 178 (1996b) 7311-7315.

[6] Giraud E., Brauman A., Keleke S., Lelong B., Raimbault M., Isolation and physiological study of an amylolytic strain of Lactobacillus plantarum. Appl. Microbiol. Biotechnol. 36 (1991) 379-383.

[7] Gold R.S., Meagher M.M., Hutkins R., Conway T., Ethanol tolerance and carbohydrate metabolism in lactobacilli, J. Indus, Microbiol. 10 (1992) 45-54.

[8] Gold R.S., Meagher M.M., Tong S., Hutkins R.W., Conway T., Cloning and expression of the Zymomonas mobilis 'production of ethanol' genes in Lactobacillus casei, Current Microbiol. 33 (1996) 256-260.

[9] Götz $F_{4}$, Elstner E.F, Sedewitz B., Lengfelder E., Oxygen utilisation by Lactobacillus plantarum. II. Superoxide and superoxide dismutation, Arch. Microbiol. 125 (1980) 215-220.

[10] Hugenholtz J., Starrenburg J.C., Diacetyl production by different strains of Lactococcus lactis subsp. lactis var diacetylactis, Appl. Microbiol. Biotechnol. 38 (1992) 17-22.

[11] Hugenholtz J., Citrate metabolism in lactic acid bacteria. F.E.M.S. Microbiol. Rev. 12 (1993) 165-217.

[12] Ingram L.O., Microbial tolerance to alcohols: role of the cell membrane. Tibtech (1986) $40-44$. 
[13] Ingram L.O., Conway T., Clark D.P., Sewell G.W., Preston J.F., Genetic engineering of ethanol production in Escherichia coli. Appl. Environ. Microbiol. 53 (1987) 2420-2425.

[14] Ingram L.O., Conway T., Expression of different levels of ethanologenic enzymes from Zymomonas mobilis in recombinant strains of Escherichia coli, Appl. Environ. Microbiol. 54 (1988) 397-404.

[15] Kok J., van der Vossen J.M.B.M., Venema G., Construction of plasmid cloning vectors for lactic streptococci which also replicate in Bacillus subtilis and Escherichia coli, Appl. Environ. Microbiol. 48 (1984) 726-731.

[16] Lerch H.P., Blöcker H., Kallwas H., Hoppe J., Tsai H., Collins J., Cloning, sequencing and expression in Escherichia coli of the D-2hydroxyisocaproate dehydrogenase gene of Lactobacillus casei, Gene 78 (1989a) 47-57.

[17] Lerch H.P., Frank R., Collins J., Cloning, sequencing and expression of the L-2hydroxyisocaproate dehydrogenase-encoding gene of Lactobacillus confusus in Escherichia coli, Gene 83 (1989b) 263-270.

[18] Marugg J.D., van Kranenburg R., Laverman P., Rutten G.A.M., de Vos W.M., Indentical transcriptional control of the divergently transcribed prtP and prtM genes that are required for proteinase production in Lactococcus lactis SK11, J. Bacteriol. 178 (1996) 1525-1531.

[19] Mat-Jan F., Alam K.Y., Clark D.P., Mutants of Escherichia coli deficient in the fermentative lactate dehydrogenase, J. Bacteriol. 171 (1989) 342-348.

[20] Ohta K., Beall D.S., Mejia J.P., Shanmugam K.T., Ingram L.O., Genetic improvement of Escherichia coli for ethanol production: chromosomal integration of Zymomonas mobilis genes encoding pyruvate decarboxylase and alcohol dehydrogenase II, Appl. Environ. Microbiol. 57 (1991) 893-900.

[21] Sneath P.H.A., Mair N.S., Sharpe M.E., Holt J.G., Bergey's manual of systematic bacteriology, Williams \&Wilkins, Baltimore, 1986.

[22] Snoep J.L., Teixeira de Mattos M.J., Starrenburg M.J.C., Hugenholtz J., Isolation, characterization, and physiological role of the pyruvate dehydrogenase complex and $\alpha$-acetolactate synthase of Lactococcus lactis subsp. lactis bv. diacetylactis, J. Bacteriol. 174 (1992) 4838-4841.

[23] Starrenburg M.J.C., Hugenholtz J., Citrate metabolism by Lactococcus and Leuconostoc spp, Appl. Environ. Microbiol. 57 (1991) 3535-3540. 